\title{
A New Birnbaum-Saunders Distribution and Its Mathematical Features Applied to Bimodal Real-World Data from Environment and Medicine
}

\author{
Jimmy Reyes ${ }^{1}\left(\mathbb{D}\right.$, Jaime Arrué ${ }^{1}\left(\mathbb{D}\right.$, Víctor Leiva ${ }^{2, *} \mathbb{D}$ and Carlos Martin-Barreiro ${ }^{3,4}(\mathbb{D})$ \\ 1 Departamento de Matemáticas, Universidad de Antofagasta, Antofagasta 1270300, Chile; \\ jimmy.reyes@uantof.cl (J.R.); jaime.arrue@uantof.cl (J.A.) \\ 2 Escuela de Ingeniería Industrial, Pontificia Universidad Católica de Valparaíso, Valparaíso 2362807, Chile \\ 3 Facultad de Ciencias Naturales y Matemáticas, Universidad Politécnica ESPOL, Guayaquil 090902, Ecuador; \\ cmmartin@espol.edu.ec \\ 4 Facultad de Ingeniería, Universidad Espíritu Santo, Samborondón 0901952, Ecuador \\ * Correspondence: victor.leiva@pucv.cl or victorleivasanchez@gmail.com
}

Citation: Reyes, J.; Arrué, J.; Leiva, V.; Martin-Barreiro, C. A New

Birnbaum-Saunders Distribution and Its Mathematical Features Applied to Bimodal Real-World Data from Environment and Medicine. Mathematics 2021, 9, 1891. https:/ / doi.org/10.3390/math9161891

Academic Editor: Francesc Pozo

Received: 6 July 2021

Accepted: 31 July 2021

Published: 9 August 2021

Publisher's Note: MDPI stays neutral with regard to jurisdictional claims in published maps and institutional affiliations.

Copyright: (c) 2021 by the authors. Licensee MDPI, Basel, Switzerland. This article is an open access article distributed under the terms and conditions of the Creative Commons Attribution (CC BY) license (https:/ / creativecommons.org/licenses/by/ $4.0 /)$.

\begin{abstract}
In this paper, we propose and derive a Birnbaum-Saunders distribution to model bimodal data. This new distribution is obtained using the product of the standard Birnbaum-Saunders distribution and a polynomial function of the fourth degree. We study the mathematical and statistical properties of the bimodal Birnbaum-Saunders distribution, including probabilistic features and moments. Inference on its parameters is conducted using the estimation methods of moments and maximum likelihood. Based on the acceptance-rejection criterion, an algorithm is proposed to generate values of a random variable that follows the new bimodal Birnbaum-Saunders distribution. We carry out a simulation study using the Monte Carlo method to assess the statistical performance of the parameter estimators. Illustrations with real-world data sets from environmental and medical sciences are provided to show applications that can be of potential use in real problems.
\end{abstract}

Keywords: Birnbaum-Saunders distribution; data science; estimation of moments and maximum likelihood; Monte Carlo method; polynomial functions; proportionate-effect law; R software

\section{Introduction}

Many phenomena may be modeled by continuous statistical distributions [1]. These distributions may be divided into symmetrical and asymmetrical with a wide variety of shapes (symmetry, skewed to the right, skewed to the left, unimodality, multimodality, platykurtosis, leptokurtosis, etc.) and supports (real numbers, positive reals, etc.). The most popular continuous distribution is the Gaussian or normal model, which has been the reference distribution for many years in statistics. However, other continuous distributions have gained space in the landscape of statistics due mainly to the important development of computation. For more details of theory, methodology, and applications of diverse continuous distributions, see [1].

A model that has received considerable attention recently is the Birnbaum-Saunders (BS) distribution, which is unimodal, asymmetric (skewed to the right), and has two parameters that modify its shape and scale. The BS distribution has good properties. For example, it is closely related to the normal distribution; it is closed under scalar multiplication (proportionality) and under reciprocation; its median coincides with the BS scale parameter; it has different shapes for its probability density function (PDF), which cover high, medium, and low asymmetry levels; it belongs to the class of log-symmetric distributions; it is a mixture model; it has mathematical arguments to be considered as a cumulative damage distribution; for more details on these and other properties, see [2-7].

After its origins in physics of materials and engineering [3], the BS distribution has been widely studied from theoretical and methodological perspectives by teams from prac- 
tically all over the world [8-17]. In addition, this model has been applied in diverse areas other than engineering by international multidisciplinary groups [7,18-25]. Among these areas, we can highlight a number of applications in environmental and medical phenomena due to the mathematical justification stated in $[7,18]$ when employing the BS distribution to model such phenomena.

Despite the wide applicability of the BS distribution, its shapes fail to model bimodal data. Note that phenomena associated with this type of data is more frequent than thought so that, under the scenario of a suitable application of the BS distribution, new bimodal models of this kind are needed to form part of the pool of statistical tools of diverse practitioners. Some BS distributions that included a bimodality parameter were introduced in $[26,27]$. However, these distributions showed little flexibility in the modeling of bimodal data. Therefore, a bimodal BS distribution that permits real-world phenomena to be modeled with high flexibility to gain precision in the information obtained from the data analysis based on such a model is needed.

The main objective of this investigation is to propose and derive a new bimodal BS distribution. Its derivation is followed by the corresponding mathematical expressions that allow the computation of probabilistic features and moments. These features enable us to obtain location, scale, variability, and shape measures, such as the mean, variance, and coefficients of variation (CV), skewness/asymmetry (CS), and kurtosis (CK). In addition, a simulation algorithm is presented, permitting us to generate values for a random variable that has the new proposed distribution. Such an algorithm allows us to simulate bimodal BS distributed data for assessing the performance of the parameter estimators of this new model. A computational implementation in the $\mathrm{R}$ software is provided, which helps to exemplify the obtained results with two real-world data sets from environmental and medical sciences and to show potential applications.

The remainder of the paper is structured in the following form: Section 2 presents background on the BS distribution and its use to model environmental and medical phenomena. In Section 3, we propose and derive the new model with its mathematical properties. In Section 4, the estimation of parameters of the bimodal BS distribution is performed. Section 5 proposes an acceptance-rejection simulation algorithm that allows us to generate bimodal BS distributed values. This section also provides the numerical results of our investigation with simulated and real data sets. At last, in Section 6, we give the conclusions, limitations, and ideas for further work that have emerged from the present study.

\section{The Birnbaum-Saunders Distribution}

In this section, we provide preliminary elements of the BS distribution. In addition, we illustrate how this distribution has mathematical arguments to describe environmental and medical data.

\subsection{Definition}

The results of this section can be seen in detail in [5]. If a random variable $Y$ has a BS distribution with parameters $\alpha$ and $\beta$, the notation $Y \sim \mathrm{BS}(\alpha, \beta)$ is employed. The BS distributed random variable $Y$ is related to the standard normal distributed random variable $V$ by means of

$$
Y=\beta\left(\frac{\alpha}{2} V+\sqrt{\left(\frac{\alpha}{2} V\right)^{2}+1}\right)^{2},
$$

where $V \sim \mathrm{N}(0,1), \alpha>0$, and $\beta>0$. The BS PDF is given by

$$
f_{Y}(y ; \alpha, \beta)=\frac{y^{-\frac{3}{2}}(y+\beta)}{2 \alpha \beta^{\frac{1}{2}}} \phi(a(y)), \quad y>0,
$$

with $a(y)=(1 / \alpha)(\sqrt{y / \beta}-\sqrt{\beta / y})$, where $\phi$ is the standard normal PDF. 


\subsection{Justifying the BS Distribution in Environmental and Medical Contexts}

The BS distribution can be adequate to model data from certain fields based on an empirical fit using goodness-of-fit techniques. However, this empirical argument by means of a simple fit can be unsatisfactory. Indeed, if we show that a distribution is suitable to model a phenomenon, employing a theoretical justification may be a better argument. For more details about the results presented below, see [5] (fatigue), [18] (environment), [7] (medicine), and [28] (neural activity).

Assume a random variable associated with a generic metric. For instance, the tumor size can result in the death of a patient in a medical setting, or in an environmental context, the accumulation of pollutants in the atmosphere leads to a critical environmental situation. A generic metric can be stated considering the effect of several independent causes that interact, which work together to increase the value of the metric over time.

When the effects of such causes are accumulated randomly, this accumulation has a Gaussian asymptotic distribution based on the central limit theorem. Nevertheless, the causes do not seem to jointly act by a simple summation. It is more natural to suppose that each cause gives an impulse, and then the effect depends on both the impulse strength and value of the metric at the moment when the impulse is working.

Let $M_{1}, \ldots, M_{k}$ be independent random variables associated with the magnitude of $k$ impulses that work sequentially according to their subscripts. Furthermore, let $O$ be a generic metric that is obtained from these impulses. Suppose that $O_{j+1}$ corresponds to value of the metric $O$ at the instant immediately before, which increases by the $(j+1)$ th impulse $M_{j+1}$ that is proportional to some function $g$ of the mentioned value of the metric $O$ stated as

$$
O_{j+1}=O_{j}+M_{j+1} g\left(O_{j}\right), \quad j=0,1, \ldots
$$

Thus, $O_{j+1}$ is the accumulated value of the metric after applying $M_{j+1}$. The formulation expressed in (2) is usually known as the proportionate-effect law. From this law, observe that the BS distribution may be reached when $g\left(O_{j}\right)=1$ in (2). Note that the BS model was built to describe the fatigue life of materials subject to cyclic stress. It causes damage that is accumulated over time by summing several small damages. If the cumulative damage is greater than the rupture value (threshold) of the material, it fails [5]. Table 1 shows a conceptual comparison for environmental, fatigue, medical, neural frameworks that follow the proportionate-effect law.

Table 1. Conceptual comparison for environmental, fatigue, medical, neural frameworks.

\begin{tabular}{lccccc}
\hline Framework & Item & Cause & Threshold & Effect & Random Variable \\
\hline Environmental & Chemical element & Contamination & Target & Emergence & Content \\
Fatigue & Material & Damage & Rupture & Failure & Fatigue life \\
Health metric & Patient & Impulse & To die & Death & Death time \\
Neural spiking & Neuron & Voltage & Firing & Spike & Inter-spike time \\
\hline
\end{tabular}

Next, we put the Frost-Dugdale differential equation in a general framework by considering

$$
\frac{\mathrm{d} o}{\mathrm{~d} m}=\frac{o S^{3}}{c}=c_{1} f\left(o^{1 / 2} b \Delta S\right) \approx c_{0}+c_{2} g(o),
$$

with $o$ being the generic metric and do its differential, $m$ being the number of impulses and $\mathrm{d} m$ its differential, $S$ being the strength exercised in each impulse, $f$ being an empirical function and $c, c_{1}$ being constants. Observe that $o^{1 / 2} b \Delta S$ stated in (3) is a strength factor related to $o$, with $b$ being an associated parameter and $\Delta S$ being the strength exercised in each impulse. Furthermore, in the approximation established in (3), $c_{0}$ is a baseline, $c_{2}$ contains other constants, and $g$ is a function of the metric $o$. Consider the elements $o, c_{0}$ and $c_{2}$ as random variables to perform a model closer to reality. 
Observe how the differential-equation stated in (3) and the proportionate-effect law given in (2) are similar. Assuming (2), apply the central limit theorem, and consider that the increment $\Delta O_{j}=O_{j+1}-O_{j}$ in the $(j+1)$ th impulse provides a small contribution to the growth of the metric. Hence, if the summation is switched by integration, $\sum_{j=1}^{k} M_{j}$ is closely a Gaussian distributed random variable, with

$$
\sum_{j=1}^{k} M_{j}=\sum_{j=1}^{k} \frac{\Delta O_{j}}{g\left(O_{j}\right)} \approx \int_{O_{s}}^{O_{k}} \frac{\mathrm{d} x}{g(x)}=\log \left(g\left(O_{k}\right)\right)-\log \left(g\left(O_{s}\right)\right)
$$

and $O_{s}$ is an initial value of the metric and $O_{k}$ its final value after applying $k$ impulses.

To generate the BS model in a general framework, consider that the mean of $O_{j}$ is $\eta$ and its variance is $Q^{2}$. Hence, a continuous extension of (4) using the time $t$ is established by

$$
I(O(t))=\int_{O\left(t_{0}\right)}^{O(t)} \frac{\mathrm{d} x}{g(x)} \sim \mathrm{N}\left(t \eta, t \varrho^{2}\right),
$$

with $O(t)$ being the value of the metric at $t$ and $O\left(t_{0}\right)$ its starting value. Now, suppose that $O_{c}>O\left(t_{0}\right)$ is a critical value of the metric at which the event of interest happens and so $T=\inf \left\{t: O(t)>O_{c}\right\}$. Thus, based on (5) and employing $\{T \leq t\} \equiv\left\{O(t)>O_{c}\right\}$, we get the cumulative distribution function (CDF) of $T$ by

$$
F_{T}(t)=\Phi\left(\frac{t \eta-I\left(O_{c}\right)}{\varrho \sqrt{t}}\right), \quad t>0 .
$$

Using (6), observe that the function $g$ in the model given in (2) establishes the dependence of the metric on its previous value. In this case, a power function for $g$ can be suitable obtaining $g(x)=x^{\varsigma}$, where $\varsigma$ is an indicator associated with the metric used for each framework (see Table 1), and $\varsigma \geq 0$, but $\varsigma \neq 1$. Hence, based on (5) and considering $g(x)=x^{\varsigma}$, we have that

$$
I(O(t))=\int_{O\left(t_{0}\right)}^{O(t)} \frac{\mathrm{d} x}{x \varsigma}=\frac{1}{\varsigma-1}\left(\frac{1}{O\left(t_{0}\right)^{\varsigma-1}}-\frac{1}{O(t)^{-1}}\right)=\frac{O\left(t_{0}\right)^{1-\varsigma}-O(t)^{1-\varsigma}}{\varsigma-1} \sim \mathrm{N}\left(t \eta, t \varrho^{2}\right) .
$$

Then, from (7)-(6) and by symmetry, we get $O(t)^{1-\varsigma} \sim \mathrm{N}\left(O_{s}^{1-\varsigma}+(1-\varsigma) t \eta,(1-\varsigma)^{2} t \varrho^{2}\right)$. Therefore, the CDF of $T$ at $t$ is expressed as

$$
F_{T}(t)= \begin{cases}\Phi\left(\frac{O_{c}^{1-\varsigma}-O_{s}^{1-\varsigma}+(\varsigma-1) t \eta}{\varsigma-1) \sqrt{t} \varrho}\right), & \text { if } \varsigma>1 ; \\ \Phi\left(\frac{O_{s}^{1-\varsigma}-O_{c}^{1-\varsigma}+(1-\varsigma) t \eta}{(1-\varsigma) \sqrt{t} \varrho}\right), & \text { if } \varsigma<1 .\end{cases}
$$

From (8), observe that the BS distribution is obtained for $\varsigma=0$. Nonetheless, if $\varsigma=1$, it is the case of the lognormal distribution $(g(x)=x)$ in the proportionate-effect law stated in (4), which cannot be obtained from (8). Hence, the CDF of $T$ formulated in (8) corresponds the BS distribution and not to the lognormal distribution.

To be specific and as an example, the setting discussed above might be placed in a framework of pollution, doing an analogy between the proportionate-effect law and a contaminant concentration model. Such an example is valid as a justification to model data on indoor and outdoor environmental quality, water contamination in lakes, groundwater, and soils, and trace metals in human tissue, blood, and feces.

To contextualize the model stated in (8) for environmental contamination, suppose the following. We assume a non-random framework. Consider that an environment has a pollutant concentration $X_{0}$ and an air mass $V_{0}$ at an initial time $t=0$. Hence, $q_{0}=X_{0} V_{0}$ corresponds to the total pollutants in the environment. If no chemical reactions are present, conservation of matter requires the total pollutant amount after dilution to be equal to 
the total pollutant amount before dilution. In this way, when $V_{1}$ is the volume of the mixture after the first dilution and $X_{1}$ the pollutant concentration in that diluted mixture, we have that

$$
q_{0}=X_{0} V_{0}=X_{1} V_{1} .
$$

Solving the equation stated in (9) for $X_{1}$, the content of $X_{1}$ is inversely proportional to the ratio of the final volume to the initial volume given by $X_{1}=\left(V_{0} / V_{1}\right) X_{0}$. Therefore, if $V_{0} / V_{1}$ is established by a dimensionless dilution $D_{1}$ that is treated as a random variable, then $X_{1}=D_{1} X_{0}$ is also a random variable, where $X_{0}$ is non-random. Such a random framework is closer to reality due to the fact that contaminants vary over time because of climatology, geography, topography, and human activity.

Let $D_{j}$ denote the $j$ th dilution. Then, the concentration $X_{2}$ in the second dilution is formulated as

$$
X_{2}=D_{2} X_{1}=D_{2} D_{1} X_{0} .
$$

In addition, let $N$ be the number of successive dilutions in a random dilution process. Then, the final concentration after $N$ dilutions is defined as

$$
X_{N}=X_{0} \prod_{j=1}^{N} D_{j}
$$

By applying the logarithm function in the expression given in (10), we get that

$$
\sum_{j=1}^{N} \log \left(D_{j}\right)=\log \left(X_{N}\right)-\log \left(X_{0}\right)=\int_{X_{0}}^{X_{N}} \frac{\mathrm{d} x}{x}
$$

Alternatively, the formulation defined in (11) may be expressed by means of a differential equation of first-order as usual in the mass balance model, after permitting the change rate of the concentration to be small enough. Once again, observe how the equations stated in (4) and (11) if $g(x)=x$ are similar. As mentioned, this formulation is associated with a lognormal distribution. Nevertheless, a more general expression for (11) might be assumed by considering

$$
\sum_{j=1}^{N} Y_{j} \approx \int_{X_{0}}^{X_{N}} \frac{\mathrm{d} x}{g(x)}
$$

Note that the expression stated in (12) should conduct a BS model upon certain conditions, with this expression being similar to (4). Such a setting permits us to consider a statistical distribution to model contaminant concentration data, which should not be symmetrical but skewed to the right.

\section{The BS Distribution under Bimodality}

In this section, we obtain the bimodal BS distribution of a random variable $Y$ based on the product of the $\mathrm{BS}(\alpha, \beta) \mathrm{PDF}$ and a fourth-degree polynomial function as a competitor of two other versions of bimodal BS distributions proposed in [26,27], which are named bimodal BS distributions of types 1 and 2, denoted by BBS1 and BBS2, respectively. The new bimodal BS distribution, which we name type 3 , is denoted by $Y \sim \operatorname{BBS} 3(\alpha, \beta, \delta)$, with $\delta \geq 0$ being the bimodality parameter.

\subsection{Probability Density Function}

A bimodal BS distribution with parameters $\alpha_{1}, \beta_{1}$, and $\delta_{1}$ of a random variable $Y$, denoted by $Y \sim \operatorname{BBS1}\left(\alpha_{1}, \beta_{1}, \delta_{1}\right)$, was postulated in [26]. This bimodal BS distribution of type 1 has a PDF stated as

$$
f_{Y}\left(y ; \alpha_{1}, \beta_{1}, \delta_{1}\right)=\frac{y^{-\frac{3}{2}}\left(y+\beta_{1}\right)}{4 \alpha_{1} \beta_{1}^{\frac{1}{2}} \Phi\left(-\delta_{1}\right)} \phi\left(|a(y)|+\delta_{1}\right), \quad y>0, \alpha_{1}>0, \beta_{1}>0,-\infty<\delta_{1}<\infty,
$$


with $a(y)$ being defined as in (1) and $\Phi$ being the standard normal CDF.

A second bimodal BS distribution with parameters $\alpha_{2}, \beta_{2}$, and $\delta_{2}$ of a random variable $Y$, denoted by $Y \sim \operatorname{BBS} 2\left(\alpha_{2}, \beta_{2}, \delta_{2}\right)$, was postulated in [27]. This bimodal BS distribution of type 2 has a PDF as a competitor of (13) given by

$$
f_{Y}\left(y ; \alpha_{2}, \beta_{2}, \delta_{2}\right)=\frac{\left(1-\delta_{2} a(y)\right)^{2}+1}{2+\delta_{2}^{2}} \phi(a(y)) \frac{y^{-\frac{3}{2}}\left(y+\beta_{2}\right)}{2 \alpha_{2} \beta_{2}^{\frac{1}{2}}}, \quad y>0, \alpha_{2}>0, \beta_{2}>0,-\infty<\delta_{2}<\infty,
$$

with $a(y)$ being defined as in (1). Next, we propose the bimodal BS distribution of type 3 as a competitor of the classical unimodal BS distribution, as well as of the bimodal BS distributions of types 1 and 2, with PDFs established in (1), (13), and (14), respectively.

Proposition 1. Let $Y \sim \operatorname{BBS} 3(\alpha, \beta, \delta)$. Then, the PDF $Y$ is stated as

$$
f_{Y}(y ; \alpha, \beta, \delta)=\frac{1+\delta\left(\frac{y-\mu}{\sigma}\right)^{4}}{1+\kappa \delta} f_{X}(y ; \alpha, \beta), \quad y>0, \alpha>0, \beta>0, \delta \geq 0,
$$

where $f_{X}$ is the PDF of $X \sim \operatorname{BS}(\alpha, \beta)$ given in (1), and

$$
\begin{aligned}
& \mu=\mathrm{E}(X)=\beta\left(1+\frac{\alpha^{2}}{2}\right), \\
& \sigma=\mathrm{SD}(X)=\sqrt{\operatorname{Var}(X)}=\alpha \beta\left(1+\frac{5 \alpha^{2}}{4}\right)^{1 / 2}, \\
& \kappa=\mathrm{CK}(X)=\frac{48+360 \alpha^{2}+633 \alpha^{4}}{\left(5 \alpha^{2}+4\right)^{2}},
\end{aligned}
$$

with SD denoting the standard deviation, $\alpha, \beta$ being shape parameters, and $\delta$ being the bimodality parameter.

Proof. Note that

$$
\begin{aligned}
\int_{0}^{\infty} f_{Y}(y ; \alpha, \beta, \delta) \mathrm{d} y & =\int_{0}^{\infty} \kappa_{0}\left(1+\delta\left(\frac{y-\mu}{\sigma}\right)^{4}\right) f_{X}(y ; \alpha, \beta) \mathrm{d} y \\
& =\kappa_{0}\left(\int_{0}^{\infty} f_{X}(y ; \alpha, \beta) \mathrm{d} y+\int_{0}^{\infty} \delta\left(\frac{y-\mu}{\sigma}\right)^{4} f_{X}(y ; \alpha, \beta) \mathrm{d} y\right) \\
& =\kappa_{0}\left(1+\delta \mathrm{E}\left(\left(\frac{Y-\mu}{\sigma}\right)^{4}\right)\right) \\
& =\kappa_{0}(1+\kappa \delta)=1,
\end{aligned}
$$

where $\kappa_{0}=1 /(1+\kappa \delta)$ and $f_{X}$ is the PDF of $X \sim \operatorname{BS}(\alpha, \beta)$.

Observe that the BBS3 distribution is obtained following the idea given in [29]. Thus, to obtain an interpretation of the modality parameter $\delta$, we present below a proposition that generalizes a classical continuous distribution allowing us to generate unimodality or bimodality in the distribution.

Proposition 2. Let $f(y ; \mu, \sigma)$ be the PDF of a continuous distribution with finite mean $\mu$, variance $\sigma^{2}$, and $C K \kappa$. Then, it is possible to build a new distribution with PDF stated as

$$
h(y ; \mu, \sigma ; \delta)=\omega(y ; \mu, \sigma, \delta) f(y ; \mu, \sigma),
$$


with $0 \leq \delta<\infty$ being the parameter that controls the unimodality or the bimodality of the distribution and where

$$
\omega(y ; \mu, \sigma, \delta)=\frac{1}{1+\kappa \delta}\left(1+\delta\left(\frac{y-\mu}{\sigma}\right)^{4}\right) .
$$

Note that the function stated in (16) is a genuine PDF.

Proof. The result is directly obtained by taking into account that $f(y ; \mu, \sigma) \geq 0$ and integrating over the support $\mathrm{R}_{Y}$ of the random variable $Y$ to have $\int_{\mathrm{R}_{Y}} f_{Y}(y) \mathrm{d} y=1$.

Observe that the parameter $\delta$ defined in the PDF given in (16) controls the unimodality or bimodality of the corresponding distribution. Additionally, by taking $\delta=0$, the parent PDF $f(y ; \mu, \sigma)$ is obtained as a special case. For a shape analysis of the BBS3 PDF compared to the BS distribution, see Figure 1.

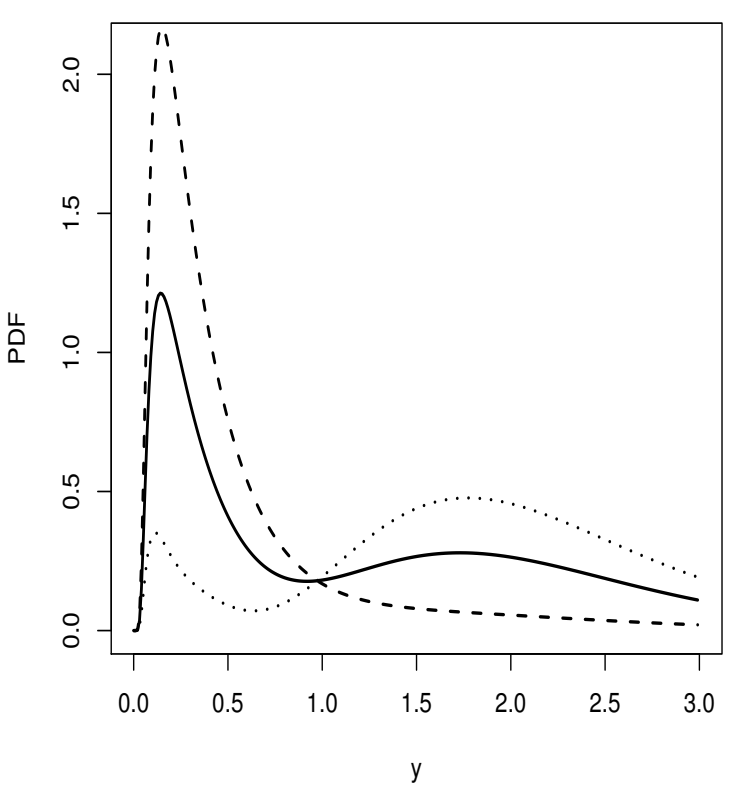

(a)

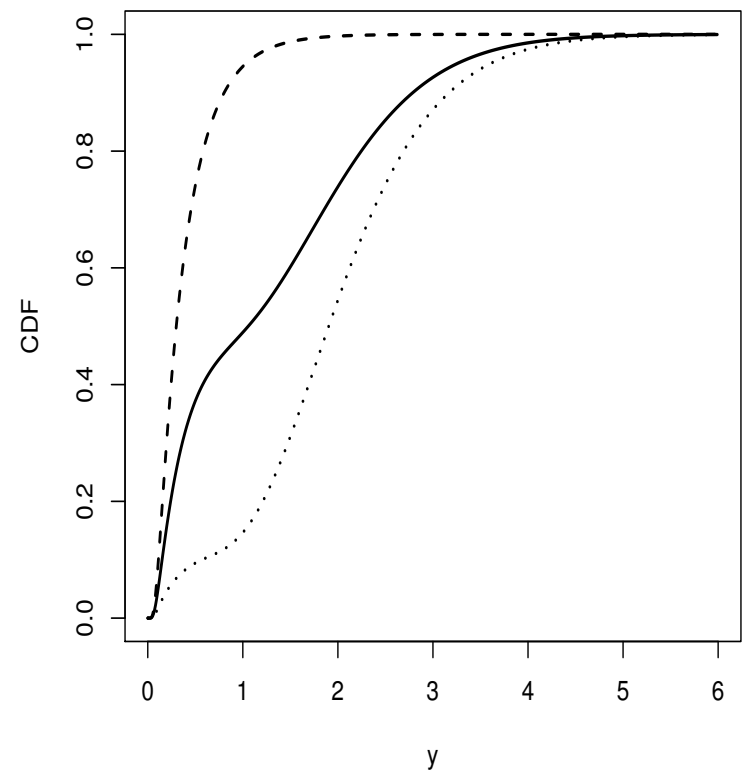

(b)

Figure 1. PDF (a) and CDF (b) plots of the BBS3 and BS distributions with $\alpha=0.8, \beta=0.3$ and $\delta=0.1$ (solid line), $\alpha=0.8$, $\beta=0.3$ and $\delta=0$ (dashed line), and $\alpha=0.8, \beta=0.3$ and $\delta=0.8$ (dotted line).

\subsection{Cumulative Distribution Function and BBS3 Properties}

Next, we give some elementary properties of the BBS3 distribution. Note that this distribution is closed under reciprocation and has the standard BS distribution as a particular case.

Proposition 3. Let $Y \sim \operatorname{BBS} 3(\alpha, \beta, \delta)$. Then, we have that

(i) $Y^{-1} \sim \operatorname{BBS3}\left(\alpha, \beta^{-1}, \delta\right)$.

(ii) If $\delta=0, Y \sim \operatorname{BS}(\alpha, \beta)$.

The following proposition states the CDF of the BBS3 distribution, which depend on the standard BS CDF. 
Proposition 4. Let $Y \sim \operatorname{BBS3}(\alpha, \beta, \delta)$. Then, the $C D F$ of $Y$ is given by

$$
\begin{aligned}
F_{Y}(t ; \alpha, \beta, \delta)=\frac{1}{1+\kappa \delta} & \left(F_{X}(t)+\frac{\delta}{2 \alpha \beta^{\frac{1}{2}} \sigma^{4} \sqrt{2 \pi}}\right. \\
& \left.\times \int_{-\infty}^{t} y^{-\frac{3}{2}}(y+\beta)(y-\mu)^{4} \mathrm{e}^{-\frac{1}{2 \alpha^{2}}\left(\sqrt{\frac{x}{\beta}}-\sqrt{\frac{\beta}{x}}\right)^{2}} \mathrm{~d} x\right),
\end{aligned}
$$

where $F_{X}$ is the $C D F$ of $X \sim \operatorname{BS}(\alpha, \beta)$, and $\mu, \sigma, \kappa$ are defined in Proposition 1.

Proof. Let $Y \sim \operatorname{BBS} 3(\alpha, \beta, \delta)$ and $F_{X}$ be the $\operatorname{CDF}$ of $X \sim \operatorname{BS}(\alpha, \beta)$. Then,

$$
\begin{aligned}
F_{Y}(t ; \alpha, \beta, \delta)= & \int_{0}^{\infty} f_{Y}(y ; \alpha, \beta, \delta) \mathrm{d} y \\
= & \frac{1}{1+\kappa \delta} \int_{-\infty}^{t}\left(1+\delta\left(\frac{y-\mu}{\sigma}\right)^{4}\right) f_{X}(y ; \alpha, \beta) \mathrm{d} y \\
= & \frac{1}{1+\kappa \delta}\left(F_{X}(t)+\frac{\delta}{2 \alpha \beta^{\frac{1}{2}} \sigma^{4} \sqrt{2 \pi}}\right. \\
& \left.\quad \times \int_{-\infty}^{t} y^{-\frac{3}{2}}(y+\beta)(y-\mu)^{4} \mathrm{e}^{-\frac{1}{2 \alpha^{2}}\left(\sqrt{\frac{x}{\beta}}-\sqrt{\frac{\beta}{x}}\right)^{2}} \mathrm{~d} x\right) .
\end{aligned}
$$

Figure 1a shows the PDFs of the BBS3 and BS distributions for different values of $\alpha, \beta$ and $\delta$. Here, we can see the bimodality of the BBS3 distribution and the unimodality of the BS distribution. Figure $1 \mathrm{~b}$ shows the graphical plots of the corresponding CDFs for different values of their parameters.

\subsection{Cumulant Generating Function}

The following proposition states the cumulant generating function of the BBS3 distribution, which depends on the moment generating function of the standard BS distribution. From the cumulant generating function, the cumulants of a distribution may be obtained, which correspond to quantities that provide an alternative to the moments of the distribution. Note that the $r$ th cumulant is computed as

$$
c_{r}=C^{(r)}(0),
$$

where $C(t)$ is the cumulant generating function of the BBS3 distribution given in the following proposition, and $C^{(r)}(0)$ defined in (17) is obtained by taking the $r$ th derivative of $C(t)$ with respect to $t$ and evaluating it at $t=0$.

Proposition 5. Let $Y \sim \operatorname{BBS} 3(\alpha, \beta, \delta)$. Then, the cumulant generating function of $Y$ is obtained as

$$
C(t)=\log \left(E\left(\mathrm{e}^{t \gamma}\right)\right), \quad-\infty<t<\infty,
$$

where

$$
\begin{aligned}
\left.\mathrm{E}\left(\mathrm{e}^{t Y}\right)\right]= & \frac{1}{1+\kappa \delta}\left(\mathrm{E}\left(\mathrm{e}^{t X}\right)\right. \\
& \left.+\frac{\delta}{2 \alpha \beta^{\frac{1}{2}} \sigma^{4} \sqrt{2 \pi}} \int_{0}^{\infty} y^{-\frac{3}{2}}(y+\beta)(y-\mu)^{4} \mathrm{e}^{-\frac{1}{2 \alpha^{2}}\left(\sqrt{\frac{y}{\beta}}-\sqrt{\frac{\beta}{y}}\right)^{2}-t y} \mathrm{~d} y\right),
\end{aligned}
$$

with $\mathrm{E}\left(\mathrm{e}^{t X}\right)$ being the moment generating function of $\mathrm{X} \sim \mathrm{BS}(\alpha, \beta)$. 
Proof.

$$
\begin{aligned}
\mathrm{E}\left(\mathrm{e}^{t Y}\right)= & \int_{0}^{\infty} \mathrm{e}^{t Y} f_{Y}(y ; \alpha, \beta, \delta) \mathrm{d} y \\
= & \frac{1}{1+\kappa \delta} \int_{-\infty}^{t}\left(1+\delta\left(\frac{y-\mu}{\sigma}\right)^{4}\right) \mathrm{e}^{t y} f_{X}(y ; \alpha, \beta) \mathrm{d} y \\
= & \frac{1}{1+\kappa \delta}\left(\mathrm{E}\left(\mathrm{e}^{t X}\right)+\frac{\delta}{2 \alpha \beta^{\frac{1}{2}} \sigma^{4} \sqrt{2 \pi}}\right. \\
& \left.\quad \times \int_{0}^{\infty} y^{-\frac{3}{2}}(y+\beta)(y-\mu)^{4} \mathrm{e}^{-\frac{1}{2 \alpha^{2}}\left(\sqrt{\frac{y}{\beta}}-\sqrt{\frac{\beta}{y}}\right)^{2}-t y} \mathrm{~d} y\right) .
\end{aligned}
$$

The $r$ th moment $\mu_{r}^{\prime}$ is an $r$ th degree polynomial function in the first $r$ cumulants, where

$$
\begin{aligned}
\mu_{1}^{\prime}= & c_{1}, \\
\mu_{2}^{\prime}= & c_{2}+c_{1}^{2} \\
\mu_{3}^{\prime}= & c_{3}+3 c_{2} c_{1}+c_{1}^{3} \\
\mu_{4}^{\prime}= & c_{4}+4 c_{3} c_{1}+3 c_{2}^{2}+6 c_{2} c_{1}^{2}+c_{1}^{4}, \\
\mu_{5}^{\prime}= & c_{5}+5 c_{4} c_{1}+10 c_{3} c_{2}+10 c_{3} c_{1}^{2}+15 c_{2}^{2} c_{1}+10 c_{2} c_{1}^{3}+c_{1}^{5}, \\
\mu_{6}^{\prime}= & c_{6}+6 c_{5} c_{1}+15 c_{4} c_{2}+15 c_{4} c_{1}^{2}+10 c_{3}^{2}+60 c_{3} c_{2} c_{1}+20 c_{3} c_{1}^{3} \\
& +15 c_{2}^{3}+45 c_{2}^{2} c_{1}^{2}+15 c_{2} c_{1}^{4}+c_{1}^{6} .
\end{aligned}
$$

Observe that the "prime" states a difference between the moments $\mu_{r}^{\prime}=\mathrm{E}\left(Y^{r}\right)$ and the central moments $\mu_{r}=\mathrm{E}\left((Y-\mu)^{r}\right)$. To obtain the central moments as functions of the cumulants, we must eliminate from these polynomials all the terms in which $c_{1}$ is a factor. Thus, from the above expressions, we generate the first six moments as $\mu_{1}=0, \mu_{2}=c_{2}$, $\mu_{3}=c_{3}, \mu_{4}=c_{4}+3 c_{2}^{2}, \mu_{5}=c_{5}+10 c_{3} c_{2}$, and $\mu_{6}=c_{6}+15 c_{4} c_{2}+10 c_{3}^{2}+15 c_{2}^{3}$. Similarly, the $r$ th cumulant $c_{r}$ is an $r$ th degree polynomial function in the first $r$ raw (non-central) moments so that the first six cumulants are stated as

$$
\begin{aligned}
c_{1}= & \mu_{1}^{\prime} \\
c_{2}= & \mu_{2}^{\prime}-\mu_{1}^{\prime 2}, \\
c_{3}= & \mu_{3}^{\prime}-3 \mu_{2}^{\prime} \mu_{1}^{\prime}+2 \mu_{1}^{\prime 3}, \\
c_{4}= & \mu_{4}^{\prime}-4 \mu_{3}^{\prime} \mu_{1}^{\prime}-3 \mu_{2}^{\prime 2}+12 \mu_{2}^{\prime} \mu_{1}^{\prime 2}-6 \mu_{1}^{\prime 4}, \\
c_{5}= & \mu_{5}^{\prime}-5 \mu_{4}^{\prime} \mu_{1}^{\prime}-10 \mu_{3}^{\prime} \mu_{2}^{\prime}+20 \mu_{3}^{\prime} \mu_{1}^{\prime 2}+30 \mu_{2}^{\prime 2} \mu_{1}^{\prime}-60 \mu_{2}^{\prime} \mu_{1}^{\prime 3}+24 \mu_{1}^{\prime 5}, \\
c_{6}= & \mu_{6}^{\prime}-6 \mu_{5}^{\prime} \mu_{1}^{\prime}-15 \mu_{4}^{\prime} \mu_{2}^{\prime}+30 \mu_{4}^{\prime} \mu_{1}^{\prime 2}-10 \mu_{3}^{\prime 2}+120 \mu_{3}^{\prime} \mu_{2}^{\prime} \mu_{1}^{\prime} \\
& -120 \mu_{3}^{\prime} \mu_{1}^{\prime 3}+30 \mu_{2}^{\prime 3}-270 \mu_{2}^{\prime 2} \mu_{1}^{\prime 2}+360 \mu_{2}^{\prime} \mu_{1}^{\prime 4}-120 \mu_{1}^{\prime 6} .
\end{aligned}
$$

To obtain the cumulants $c_{r}$ for $r>1$ as functions of the central moments, we must remove from these polynomials all the terms in which $\mu_{1}^{\prime}$ is as a factor. Hence, from the above expressions, we generate the first six cumulants as $c_{2}=\mu_{2}, c_{3}=\mu_{3}, c_{4}=\mu_{4}-3 \mu_{2}{ }^{2}$, $c_{5}=\mu_{5}-10 \mu_{3} \mu_{2}$, and $c_{6}=\mu_{6}-15 \mu_{4} \mu_{2}-10 \mu_{3}{ }^{2}+30 \mu_{2}{ }^{3}$. This indicates that the first cumulant is the mean, the second cumulant is the variance, and the third cumulant is the third central moment. However, from the fourth cumulant, they are not equal to the central moments.

\subsection{Moments}

The following proposition states the moments of the BBS3 distribution. Essentially, these moments depend on the moments of the standard BS distribution. 
Proposition 6. Let $Y \sim \operatorname{BBS3}(\alpha, \beta, \delta)$. Then, for $r=1,2, \ldots$, we have that

$$
\begin{aligned}
\mu_{r}= & \mathrm{E}\left(Y^{r}\right)=\frac{1}{1+\kappa \delta}\left(\left(1+\frac{\delta \mu^{4}}{\sigma^{4}}\right) \mathrm{E}\left(X^{r}\right)\right. \\
& \left.+\frac{\delta}{\sigma^{4}}\left(\mathrm{E}\left(X^{r+4}\right)-4 \mu \mathrm{E}\left(X^{r+3}\right)+6 \mu^{2} \mathrm{E}\left(X^{r+2}\right)-4 \mu^{3} \mathrm{E}\left(X^{r+1}\right)\right)\right),
\end{aligned}
$$

where $\mathrm{E}\left(X^{r}\right)$ is the rth moment of $X \sim \mathrm{BS}(\alpha, \beta)$, and $\mu, \sigma, \kappa$ are given in Proposition 1.

Proof. Let $Y \sim \operatorname{BBS} 3(\alpha, \beta, \delta)$ and $F_{X}$ be the $\operatorname{CDF}$ of $X \sim \operatorname{BS}(\alpha, \beta)$. Then,

$$
\begin{aligned}
f_{Y}(y ; \alpha, \beta, \delta) & =\frac{1+\delta\left(\frac{y-\mu}{\sigma}\right)^{4}}{1+\kappa \delta} f_{X}(y ; \alpha, \beta) \\
& =\frac{1}{1+\kappa \delta}\left(1+\frac{\delta}{\sigma^{4}}(y-\mu)^{4}\right) f_{X}(y ; \alpha, \beta) \\
& =\frac{1}{1+\kappa \delta}\left(1+\frac{\delta}{\sigma^{4}}\left(y^{4}-4 \mu y^{3}+6 \mu^{2} y^{2}-4 \mu^{3} y+\mu^{4}\right)\right) f_{X}(y ; \alpha, \beta) .
\end{aligned}
$$

Thus, we have that

$$
\begin{aligned}
\mu_{r}= & \mathrm{E}\left(Y^{r}\right) \\
= & \frac{1}{1+\kappa \delta} \int_{0}^{\infty} y^{r}\left(1+\frac{\delta}{\sigma^{4}}\left(y^{4}-4 \mu y^{3}+6 \mu^{2} y^{2}-4 \mu^{3} y+\mu^{4}\right)\right) f_{X}(y ; \alpha, \beta) \mathrm{d} y \\
= & \frac{1}{1+\kappa \delta}\left(\left(1+\frac{\delta \mu^{4}}{\sigma^{4}}\right) \mathrm{E}\left(X^{r}\right)\right. \\
& \left.\quad+\frac{\delta}{\sigma^{4}}\left(\mathrm{E}\left(X^{r+4}\right)-4 \mu \mathrm{E}\left(X^{r+3}\right)+6 \mu^{2} \mathrm{E}\left(X^{r+2}\right)-4 \mu^{3} \mathrm{E}\left(X^{r+1}\right)\right)\right) .
\end{aligned}
$$

Observe that the six first moments of $X \sim \mathrm{BS}(\alpha, \beta)$ are given by

$$
\begin{aligned}
\mathrm{E}(X) & =\frac{1}{2} \beta\left(\alpha^{2}+2\right) \\
\mathrm{E}\left(X^{2}\right) & =\frac{1}{2} \beta^{2}\left(3 \alpha^{4}+4 \alpha^{2}+2\right) \\
\mathrm{E}\left(X^{3}\right) & =\frac{1}{2} \beta^{3}\left(15 \alpha^{6}+18 \alpha^{4}+9 \alpha^{2}+2\right) \\
\mathrm{E}\left(X^{4}\right) & =\frac{1}{2} \beta^{4}\left(105 \alpha^{8}+120 \alpha^{6}+60 \alpha^{4}+16 \alpha^{2}+2\right) \\
\mathrm{E}\left(X^{5}\right) & =\frac{\beta^{5}}{2}\left(945 \alpha^{10}+1050 \alpha^{8}+525 \alpha^{6}+150 \alpha^{4}+25 \alpha^{2}+2\right) \\
\mathrm{E}\left(X^{6}\right) & =\frac{\beta^{6}}{2}\left(10,395 \alpha^{12}+11,340 \alpha^{10}+5670 \alpha^{8}+1680 \alpha^{6}+315 \alpha^{4}+36 \alpha^{2}+2\right)
\end{aligned}
$$

which allow us to obtain the corresponding moments of $Y \sim \operatorname{BBS} 3(\alpha, \beta, \delta)$ from Proposition 6.

\section{Inference}

In this section, we discuss statistical inference based on the moment and maximum likelihood estimators of the BBS3 distribution parameters. For more details about moment estimation for the standard BS distribution, see [12,30]. 


\subsection{Moment Estimators}

Let $Y=\left(Y_{1}, \ldots, Y_{n}\right)^{\top}$ be a random sample of size $n$ from $Y \sim \operatorname{BBS3}(\boldsymbol{\theta})$, with $\boldsymbol{\theta}=(\alpha, \beta, \delta)$, and $\boldsymbol{y}=\left(y_{1}, \ldots, y_{n}\right)^{\top}$ the observed value of $\boldsymbol{Y}$. Then, the moment estimator of $\boldsymbol{\theta}$ can be obtained from the equations stated as

$$
\begin{gathered}
\bar{y}=\frac{1}{1+\kappa \delta}\left(\left(1+\frac{\delta \mu^{4}}{\sigma^{4}}\right) \mathrm{E}(X)+\frac{\delta}{\sigma^{4}}\left\{\mathrm{E}\left(X^{5}\right)-4 \mu \mathrm{E}\left(X^{4}\right)+6 \mu^{2} \mathrm{E}\left(X^{3}\right)-4 \mu^{3} \mathrm{E}\left(X^{2}\right)\right)\right) \\
\overline{y^{2}}=\frac{1}{1+\kappa \delta}\left(\left(1+\frac{\delta \mu^{4}}{\sigma^{4}}\right) \mathrm{E}\left(X^{2}\right)+\frac{\delta}{\sigma^{4}}\left(\mathrm{E}\left(X^{6}\right)-4 \mu \mathrm{E}\left(X^{5}\right)+6 \mu^{2} \mathrm{E}\left(X^{4}\right)-4 \mu^{3} \mathrm{E}\left(X^{3}\right)\right)\right), \\
\overline{y^{3}}=\frac{1}{1+\kappa \delta}\left(\left(1+\frac{\delta \mu^{4}}{\sigma^{4}}\right) \mathrm{E}\left(X^{3}\right)+\frac{\delta}{\sigma^{4}}\left(\mathrm{E}\left(X^{7}\right)-4 \mu \mathrm{E}\left(X^{6}\right)+6 \mu^{2} \mathrm{E}\left(X^{5}\right)-4 \mu^{3} \mathrm{E}\left(X^{4}\right)\right)\right), \\
\text { where } \overline{y^{r}}=\sum_{i=1}^{n} y_{i}^{r} / n, \text { for } r=1,2,3 .
\end{gathered}
$$

\subsection{Maximum Likelihood Estimators}

Let $Y=\left(Y_{1}, \ldots, Y_{n}\right)^{\top}$ be a random sample of size $n$ from $Y \sim \operatorname{BBS3}(\boldsymbol{\theta})$, with $\boldsymbol{\theta}=(\alpha, \beta, \delta)$, and $\boldsymbol{y}=\left(y_{1}, \ldots, y_{n}\right)^{\top}$ the observed value of $\boldsymbol{Y}$. Then, the maximum likelihood estimator of $\boldsymbol{\theta}$ can be obtained from the corresponding log-likelihood function defined as

$$
\begin{aligned}
l(\theta ; y) \propto & \sum_{i=1}^{n} \log \left(1+\delta z_{i}^{4}\right)-n \log (1+\kappa \delta)-\sum_{i=1}^{n} \frac{a^{2}\left(y_{i}\right)}{2}-\frac{3}{2} \sum_{i=1}^{n} \log \left(y_{i}\right)+\sum_{i=1}^{n} \log \left(y_{i}+\beta\right) \\
& -n \log (\alpha)-\frac{n}{2} \log (\beta),
\end{aligned}
$$

where $z_{i}=\left(y_{i}-\mu\right) / \sigma$, for $i=1, \ldots, n$. Thus, the score vector associated with the loglikelihood function stated in (18) has elements given by

$$
\begin{aligned}
S_{\alpha}= & \sum_{i=1}^{n} \frac{\alpha \sigma^{2}-\delta z_{i}^{3}\left(5 z_{i} \alpha^{4} \beta^{2}+4 \alpha^{2} \sigma \beta+4 z_{i} \sigma^{2}\right)}{\alpha \sigma^{2}\left(1+\delta z_{i}^{4}\right)}-\frac{n \delta \alpha^{3} \beta^{2}\left(2532 \alpha^{4} \beta^{2}+732 \alpha^{2} \beta^{2}-5 \kappa \sigma^{2}\right)}{\sigma^{4}(1+\kappa \delta)} \\
& +\frac{1}{\alpha} \sum_{i=1}^{n} a\left(y_{i}\right)^{2}-\frac{n}{\alpha} \\
S_{\beta}= & \sum_{i=1}^{n} \frac{2 \sigma \beta-\delta\left(\beta \alpha^{2}+2 \beta+2 z_{i} \sigma\right)}{2 \sigma \beta\left(1+\delta z_{i}^{4}\right)}+\frac{1}{2 \alpha^{2} \beta} \sum_{i=1}^{n}\left(\frac{y_{i}}{\beta}-\frac{\beta}{y_{i}}\right)+\sum_{i=1}^{n} \frac{1}{y_{i}+\beta}-\frac{n}{2 \beta^{\prime}}, \\
S_{\delta}= & \sum_{i=1}^{n} \frac{z_{i}^{4}}{\left(1+\delta z_{i}^{4}\right)}-\frac{n \kappa}{(1+\kappa \delta)} .
\end{aligned}
$$

By solving the estimation equations obtained from the score vector numerically, we obtain maximum likelihood estimators of the parameters $\alpha, \beta$ and $\delta$.

\section{Numerical Applications}

In this section, we use simulated and real-world data to show our numerical results. An algorithm is employed to simulate bimodal BS distributed data and then evaluate the performance of the estimators of the new bimodal BS distribution parameters. Hence, a computational implementation in the $\mathrm{R}$ software produced by the authors of this study is utilized to illustrate the obtained results with real-world data sets from environmental and medical sciences to show potential applications. The data analyzed in this paper and the computational codes are available from the authors under request.

\subsection{Simulation Algorithm and Computer Characteristics}

Even though some software provide built-in random number generators, there are probability distributions that are not covered by these software. This is the case of the 
BBS3 distribution. We use the acceptance-rejection method to generate random numbers from the $\operatorname{BBS3}(\alpha, \beta, \delta)$ distribution with PDF defined in (15) according to Algorithm 1. The results in a sequence of $n$ random numbers are stored within an array that we name $n \_$vector. Since the BBS3 distribution has a non-finite support, we use a constant $l_{1}>0$ to limit the BBS3 values generated. Furthermore, we employ a constant $l_{2}>0$ corresponding to the maximum value of the $\operatorname{BBS} 3 \operatorname{PDF} f$, which must be evaluated at the true parameters. In addition to the parameters $\alpha, \beta, \gamma$ of the BBS3 distribution, to build the algorithm, we need to define:

- $\quad n$ : The length of the $n \_$vector.

- $\quad Y$ : A random variable with $\operatorname{BBS3}(\alpha, \beta, \delta)$ distribution.

- $f_{Y}(y)$ : The BBS3 PDF with $y>0$.

- $\quad l_{1}$ : A lower limit for the BBS3 numbers to be generated with $l_{1}>0$.

- $\quad l_{2}$ : The maximum value of $f_{Y}$ with $l_{2}>0$.

- $U_{1}$ : A random variable with a uniform distribution in $\left(0, l_{1}\right), \mathrm{U}\left(0, l_{1}\right)$ in short.

- $U_{2}$ : A random variable with a $\mathrm{U}\left(0, l_{2}\right)$ distribution.

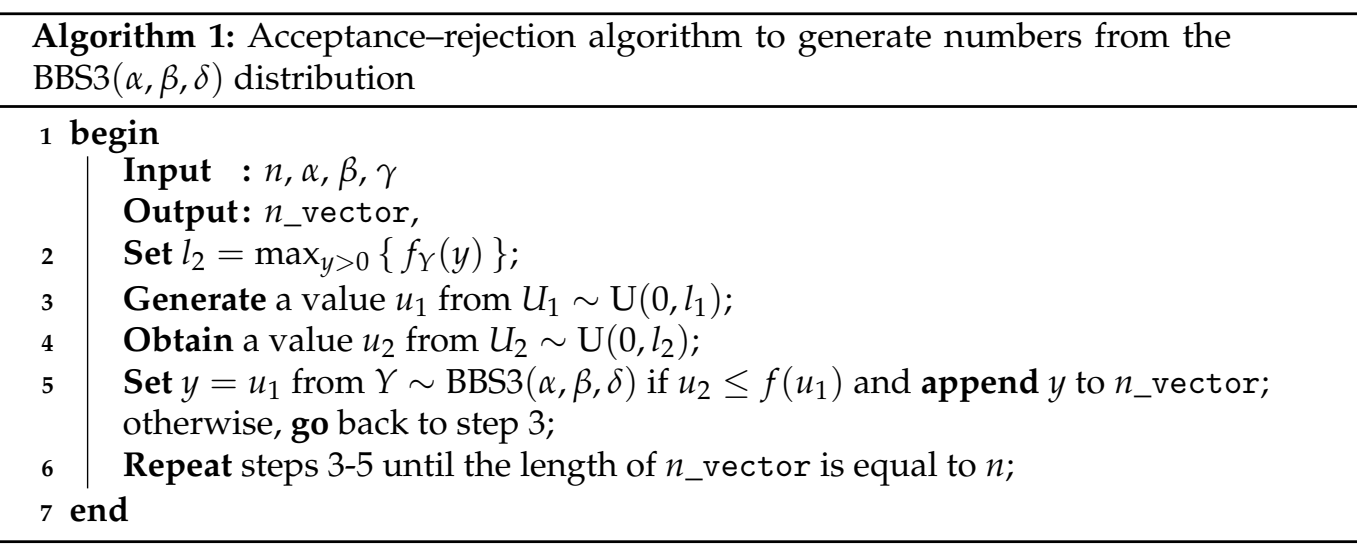

The computational experiments were carried out in the R programming language, using a computer with the following characteristics: (i) OS: Windows 10 Pro for 64 bits; (ii) RAM: 8 Gigabytes; and (iii) Processor: Intel(R) Core(TM) i7-8550U CPU @ 1.99 GigaHertz. Algorithm 1 was executed 100 times with $n=100,000$, and an average processing time equal to 2.9 seconds was obtained. When executing this algorithm 5000 times, with $n=50,200$, the average processing times were 0.035 and 0.039 seconds, respectively.

\subsection{Results of the Simulation Study}

We use Algorithm 1 to conduct a Monte Carlo simulation study. The results of this study are reported in Table 2 to assess the behavior of the maximum likelihood estimators based on 5000 generated samples of sizes $n=50,100,150,200$ from a BBS3 $(\alpha, \beta, \delta)$ distributed population. For each sample generated, maximum likelihood estimates were computed numerically using a Newton-Raphson procedure. Then, empirical means, SDs, average length (ALI) and covering probabilities (C) of $95 \%$ confidence intervals are reported. Note that the empirical bias becomes smaller as the sample size $n$ increases, as expected. Table 3 shows the results of a simulation with 5000 samples but with smaller sizes $(n=10,25,40)$ and using moment estimation.

\subsection{Illustrative Example I with Real-World Data}

The first data set corresponds to the duration of the eruption for the Old Faithful geyser in Yellowstone National Park, Wyoming, USA; the data were reported in [31]. Descriptive statistics for the data set are the following: 272 (sample size), 43 (minimum), 96 (maximum), 76 (median), 70.897 (mean), 13.595 (SD), 19.176 (CV), -0.414 (CS) and 1.844 (CK). Table 4 reports the maximum likelihood estimates, standard errors (SEs), and log-likelihood (loglik) values for the BBS1, BBS2, and BBS3 models. Furthermore, we report the values of 
the Akaike (AIC) and Bayesian information (BIC) criteria as well as the $p$-values of the Kolmogorov-Smirnov (KS) test. Figure 2a shows the histogram with estimated PDFs, which indicates that the BBS3 model fits the data better than the BBS1 and BBS2 models. This result is supported by Figure $2 \mathrm{~b}$ with the estimated CDF and by Figure 3 based on the theoretical versus empirical quantiles $(\mathrm{QQ})$ plots.

Table 2. Empirical means, SDs, ALIs, and covering probabilities of $95 \%$ confidence intervals based on simulated data from the $\operatorname{BBS} 3(\alpha, \beta, \delta)$ distribution for its parameter estimators using the maximum likelihood method.

\begin{tabular}{|c|c|c|c|c|c|c|c|c|c|c|c|c|c|c|c|}
\hline$n$ & $\alpha$ & $\beta$ & $\delta$ & $\operatorname{Mean}(\widehat{\alpha})$ & $\operatorname{SD}(\widehat{\alpha})$ & $\operatorname{ALI}(\widehat{\alpha})$ & $\mathrm{C}(\widehat{\alpha})$ & $\operatorname{Mean}(\widehat{\beta})$ & $\operatorname{SD}(\widehat{\beta})$ & $\operatorname{ALI}(\widehat{\beta})$ & $\mathrm{C}(\widehat{\beta})$ & $\operatorname{Mean}(\widehat{\delta})$ & $\operatorname{SD}(\widehat{\delta})$ & $\operatorname{ALI}(\widehat{\delta})$ & $\mathrm{C}(\widehat{\delta})$ \\
\hline 50 & 0.1 & 0.2 & 0.8 & 0.0992 & 0.0067 & 0.0261 & 94.90 & 0.2003 & 0.0036 & 0.0143 & 94.80 & 1.0105 & 0.5855 & 2.2953 & 93.44 \\
\hline 100 & 0.1 & 0.2 & 0.8 & 0.0995 & 0.0047 & 0.0183 & 95.22 & 0.2001 & 0.0024 & 0.0093 & 95.04 & 0.8951 & 0.3361 & 1.3174 & 94.30 \\
\hline 150 & 0.1 & 0.2 & 0.8 & 0.0998 & 0.0038 & 0.0148 & 95.00 & 0.2001 & 0.0020 & 0.0078 & 95.34 & 0.8505 & 0.2367 & 0.9278 & 94.36 \\
\hline 200 & 0.1 & 0.2 & 0.8 & 0.0998 & 0.0033 & 0.0128 & 95.06 & 0.2001 & 0.0017 & 0.0066 & 94.98 & 0.8489 & 0.2089 & 0.8188 & 94.64 \\
\hline 50 & 0.2 & 0.3 & 0.5 & 0.1974 & 0.0165 & 0.0646 & 94.36 & 0.3019 & 0.0138 & 0.0541 & 94.70 & 0.6083 & 0.3473 & 1.3615 & 94.18 \\
\hline 100 & 0.2 & 0.3 & 0.5 & 0.1983 & 0.0114 & 0.0446 & 94.58 & 0.3014 & 0.0094 & 0.0369 & 94.48 & 0.5549 & 0.1918 & 0.7518 & 93.60 \\
\hline 150 & 0.2 & 0.3 & 0.5 & 0.1991 & 0.0095 & 0.0371 & 94.46 & 0.3006 & 0.0076 & 0.0299 & 94.94 & 0.5307 & 0.1422 & 0.5576 & 94.36 \\
\hline 200 & 0.2 & 0.3 & 0.5 & 0.1994 & 0.0083 & 0.0325 & 94.72 & 0.3005 & 0.0067 & 0.0262 & 94.76 & 0.5212 & 0.1203 & 0.4714 & 95.16 \\
\hline 50 & 0.5 & 0.5 & 0.5 & 0.4890 & 0.0601 & 0.2357 & 95.08 & 0.5220 & 0.0829 & 0.3248 & 93.90 & 0.6472 & 0.4457 & 1.7472 & 94.56 \\
\hline 100 & 0.5 & 0.5 & 0.5 & 0.4947 & 0.0395 & 0.1550 & 95.06 & 0.5102 & 0.0537 & 0.2105 & 94.20 & 0.5576 & 0.2155 & 0.8447 & 94.28 \\
\hline 150 & 0.5 & 0.5 & 0.5 & 0.4965 & 0.0323 & 0.1267 & 94.92 & 0.5067 & 0.0431 & 0.1690 & 94.64 & 0.5322 & 0.1494 & 0.5857 & 94.02 \\
\hline 200 & 0.5 & 0.5 & 0.5 & 0.4971 & 0.0273 & 0.1071 & 94.56 & 0.5050 & 0.0365 & 0.1429 & 94.56 & 0.5233 & 0.1263 & 0.4951 & 94.32 \\
\hline 50 & 0.5 & 0.8 & 0.2 & 0.4939 & 0.0439 & 0.1722 & 94.96 & 0.8191 & 0.0957 & 0.3751 & 94.26 & 0.2211 & 0.0969 & 0.3797 & 94.96 \\
\hline 100 & 0.5 & 0.8 & 0.2 & 0.4978 & 0.0301 & 0.1179 & 94.90 & 0.8079 & 0.0643 & 0.2520 & 94.68 & 0.2075 & 0.0547 & 0.2143 & 94.86 \\
\hline 150 & 0.5 & 0.8 & 0.2 & 0.4980 & 0.0245 & 0.0960 & 94.62 & 0.8061 & 0.0516 & 0.2024 & 94.56 & 0.2051 & 0.0440 & 0.1723 & 94.98 \\
\hline 200 & 0.5 & 0.8 & 0.2 & 0.4986 & 0.0216 & 0.0846 & 95.12 & 0.8053 & 0.0451 & 0.1767 & 94.96 & 0.2035 & 0.0380 & 0.1492 & 95.04 \\
\hline 50 & 0.5 & 0.8 & 0.4 & 0.4898 & 0.0560 & 0.2195 & 94.94 & 0.8309 & 0.1239 & 0.4858 & 93.92 & 0.4934 & 0.3101 & 1.2156 & 95.00 \\
\hline 100 & 0.5 & 0.8 & 0.4 & 0.4955 & 0.0374 & 0.1466 & 94.30 & 0.8139 & 0.0823 & 0.3226 & 94.70 & 0.4366 & 0.1479 & 0.5797 & 94.48 \\
\hline 150 & 0.5 & 0.8 & 0.4 & 0.4970 & 0.0305 & 0.1196 & 94.62 & 0.8100 & 0.0652 & 0.2558 & 94.76 & 0.4199 & 0.1089 & 0.4269 & 94.42 \\
\hline 200 & 0.5 & 0.8 & 0.4 & 0.4975 & 0.0258 & 0.1011 & 94.74 & 0.8073 & 0.0554 & 0.2170 & 94.68 & 0.4151 & 0.0908 & 0.3559 & 94.52 \\
\hline 50 & 0.8 & 0.8 & 0.1 & 0.7923 & 0.0670 & 0.2626 & 94.82 & 0.8212 & 0.1195 & 0.4685 & 94.62 & 0.1091 & 0.0397 & 0.1557 & 93.66 \\
\hline 100 & 0.8 & 0.8 & 0.1 & 0.7961 & 0.0478 & 0.1872 & 94.68 & 0.8116 & 0.0830 & 0.3254 & 94.88 & 0.1037 & 0.0251 & 0.0983 & 94.82 \\
\hline 150 & 0.8 & 0.8 & 0.1 & 0.7971 & 0.0386 & 0.1513 & 95.06 & 0.8079 & 0.0663 & 0.2600 & 94.62 & 0.1026 & 0.0200 & 0.0783 & 95.00 \\
\hline 200 & 0.8 & 0.8 & 0.1 & 0.7971 & 0.0333 & 0.1303 & 94.80 & 0.8065 & 0.0564 & 0.2210 & 94.82 & 0.1023 & 0.0170 & 0.0667 & 94.68 \\
\hline
\end{tabular}

Table 3. Empirical means, SDs, ALIs, and covering probabilities of $95 \%$ confidence intervals based on simulated data from the $\operatorname{BBS3}(\alpha, \beta, \delta)$ distribution for its parameter estimators using the moment method.

\begin{tabular}{|c|c|c|c|c|c|c|c|c|c|c|c|c|c|c|c|}
\hline$n$ & $\alpha$ & $\beta$ & $\delta$ & $\operatorname{Mean}(\widehat{\alpha})$ & $\operatorname{SD}(\widehat{\alpha})$ & $\operatorname{ALI}(\widehat{\alpha})$ & $\mathrm{C}(\widehat{\alpha})$ & $\operatorname{Mean}(\widehat{\beta})$ & $\operatorname{SD}(\widehat{\beta})$ & $\operatorname{ALI}(\widehat{\beta})$ & $\mathrm{C}(\widehat{\beta})$ & $\operatorname{Mean}(\widehat{\delta})$ & $\operatorname{SD}(\widehat{\delta})$ & $\operatorname{ALI}(\widehat{\delta})$ & $\mathrm{C}(\widehat{\delta})$ \\
\hline 10 & 0.1 & 0.2 & 0.8 & 0.0928 & 0.0233 & 0.0912 & 97.44 & 0.2014 & 0.0143 & 0.0559 & 94.94 & 0.8100 & 0.0722 & 0.2829 & 95.06 \\
\hline 25 & 0.1 & 0.2 & 0.8 & 0.0973 & 0.0123 & 0.0482 & 96.16 & 0.2007 & 0.0091 & 0.0357 & 95.02 & 0.8073 & 0.0624 & 0.2447 & 95.70 \\
\hline 40 & 0.1 & 0.2 & 0.8 & 0.0984 & 0.0091 & 0.0357 & 94.88 & 0.2005 & 0.0074 & 0.0289 & 95.30 & 0.8044 & 0.0528 & 0.2070 & 96.56 \\
\hline 10 & 0.2 & 0.3 & 0.5 & 0.1873 & 0.0491 & 0.1927 & 94.76 & 0.3094 & 0.0495 & 0.1939 & 94.88 & 0.5334 & 0.1666 & 0.6532 & 95.62 \\
\hline 25 & 0.2 & 0.3 & 0.5 & 0.1953 & 0.0313 & 0.1228 & 95.10 & 0.3035 & 0.0309 & 0.1210 & 94.82 & 0.5232 & 0.1346 & 0.5276 & 95.08 \\
\hline 40 & 0.2 & 0.3 & 0.5 & 0.1970 & 0.0249 & 0.0978 & 95.24 & 0.3025 & 0.0244 & 0.0956 & 94.88 & 0.5193 & 0.1175 & 0.4604 & 95.30 \\
\hline 10 & 0.5 & 0.8 & 0.4 & 0.4925 & 0.0509 & 0.1996 & 98.22 & 0.7449 & 0.1350 & 0.5291 & 96.18 & 0.1278 & 1.3174 & 5.1644 & 98.66 \\
\hline 25 & 0.5 & 0.8 & 0.4 & 0.4963 & 0.0482 & 0.1890 & 99.04 & 0.7608 & 0.1079 & 0.4231 & 93.90 & 0.1715 & 1.1857 & 4.6480 & 98.82 \\
\hline 40 & 0.5 & 0.8 & 0.4 & 0.4973 & 0.0461 & 0.1806 & 98.98 & 0.7675 & 0.0948 & 0.3717 & 90.64 & 0.2090 & 0.8960 & 3.5124 & 97.96 \\
\hline 10 & 0.5 & 0.5 & 0.5 & 0.5017 & 0.0509 & 0.1995 & 98.06 & 0.4818 & 0.0842 & 0.3302 & 90.06 & 0.4354 & 0.4059 & 1.5909 & 88.36 \\
\hline 25 & 0.5 & 0.5 & 0.5 & 0.4994 & 0.0327 & 0.1282 & 98.38 & 0.4961 & 0.0466 & 0.1825 & 96.06 & 0.4963 & 0.2398 & 0.9402 & 95.68 \\
\hline 40 & 0.5 & 0.5 & 0.5 & 0.4996 & 0.0171 & 0.0670 & 95.84 & 0.4986 & 0.0336 & 0.1316 & 96.50 & 0.5030 & 0.1781 & 0.6980 & 96.34 \\
\hline
\end{tabular}


Table 4. Maximum likelihood estimates (and SEs in parenthesis) of the indicated distribution parameters with eruption data and model selection indicators.

\begin{tabular}{crrr}
\hline Estimate/Indicator & BBS1 (SE) & BBS2 (SE) & BBS3 (SE) \\
\hline$\widehat{\alpha}$ & $0.0898(0.0045)$ & $0.1257(0.0039)$ & $0.1042(0.0027)$ \\
$\widehat{\beta}$ & $65.0852(0.3740)$ & $66.9851(0.4785)$ & $63.8997(0.4378)$ \\
$\widehat{\delta}$ & $-2.1242(0.1392)$ & $-3.8569(0.0225)$ & $1.7480(0.4438)$ \\
Log-likelihood & -1054.180 & -1050.573 & -1036.824 \\
AIC & 2114.360 & 2107.146 & 2079.648 \\
BIC & 2129.325 & 2122.111 & 2090.465 \\
KS statistic & 0.1764 & 0.0808 & 0.0735 \\
KS $p$-value & 0.0004 & 0.3359 & 0.454 \\
\hline
\end{tabular}

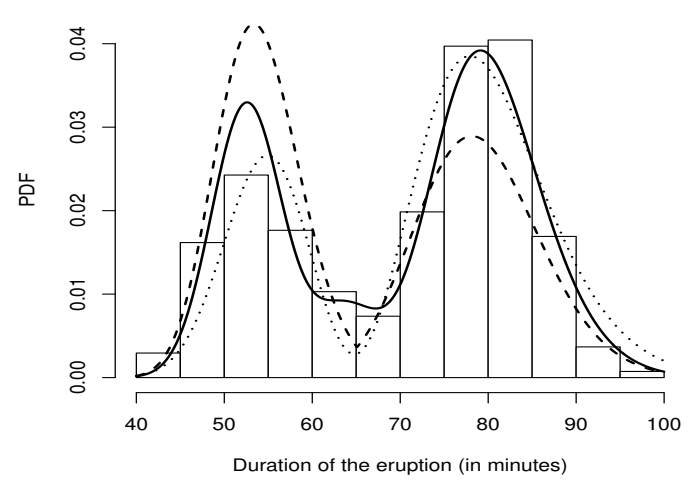

(a)

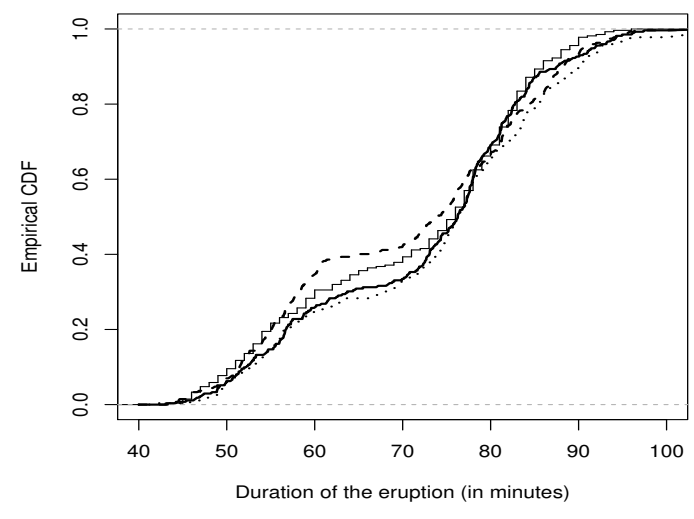

(b)

Figure 2. Histogram with estimated BBS1—dotted line, BBS2 - dashed line, and BBS3 — solid line, PDFs (a); and plots of empirical CDF versus the BBS1-dotted line, BBS2-dashed line, and BBS3-solid line, theoretical CDFs (b) with eruption data.

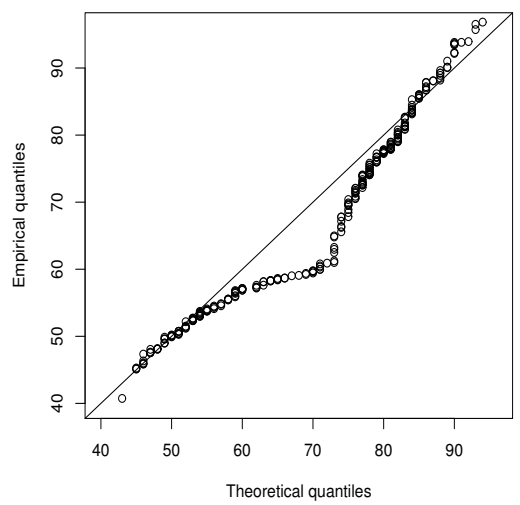

(a)

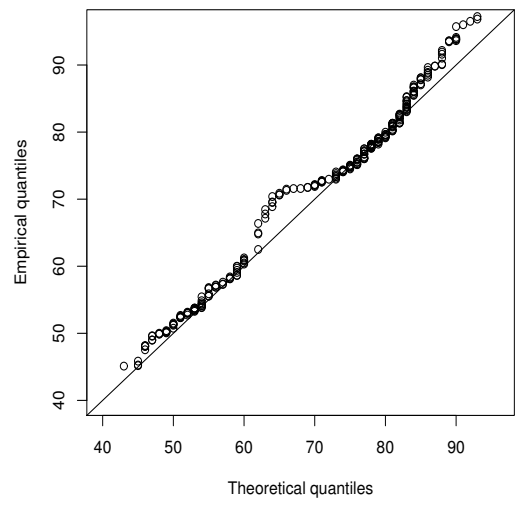

(b)

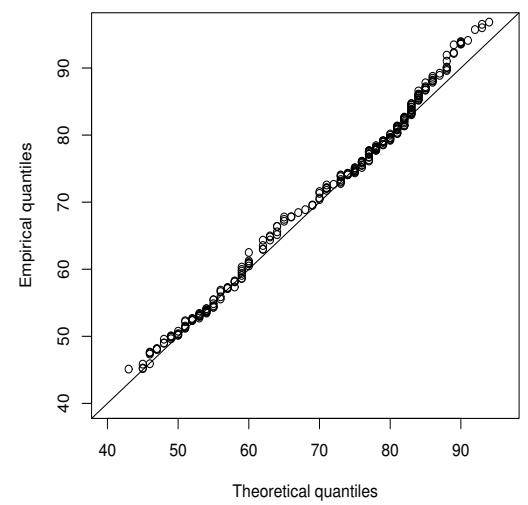

(c)

Figure 3. QQ plots of the BBS1 (a), BBS2 (b) and BBS3 (c) distributions with eruption data.

Once the best model to describe these data has been selected, that is, the BBS3 distribution, and its parameters estimated, diverse statistical analyses can be conducted by practitioners and users of statistics. For example, by estimating the mean duration of the eruption of geysers, certain measures may be established to reduce the risk against people visiting the Yellowstone National Park in Wyoming, USA. In our case, the mean duration of the eruption of geysers in Yellowstone Park is 49.205 minutes. This information is 
obtained from the estimates reported in Table 4 and the expressions taken from Section 3.4 specified as

$$
\mu=\mathrm{E}(Y)=\frac{1}{1+\kappa \delta}\left(\left(1+\frac{\delta \mu^{4}}{\sigma^{4}}\right) \mathrm{E}\left(X^{r}\right)+\frac{\delta}{\sigma^{4}}\left(\mathrm{E}\left(X^{5}\right)-4 \mu \mathrm{E}\left(X^{4}\right)+6 \mu^{2} \mathrm{E}\left(X^{3}\right)-4 \mu^{3} \mathrm{E}\left(X^{2}\right)\right)\right),
$$

where

$$
\begin{aligned}
\mathrm{E}(X) & =\frac{1}{2} \beta\left(\alpha^{2}+2\right) \\
\mathrm{E}\left(X^{2}\right) & =\frac{1}{2} \beta^{2}\left(3 \alpha^{4}+4 \alpha^{2}+2\right) \\
\mathrm{E}\left(X^{3}\right) & =\frac{1}{2} \beta^{3}\left(15 \alpha^{6}+18 \alpha^{4}+9 \alpha^{2}+2\right) \\
\mathrm{E}\left(X^{4}\right) & =\frac{1}{2} \beta^{4}\left(105 \alpha^{8}+120 \alpha^{6}+60 \alpha^{4}+16 \alpha^{2}+2\right) \\
\mathrm{E}\left(X^{5}\right) & =\frac{\beta^{5}}{2}\left(945 \alpha^{10}+1050 \alpha^{8}+525 \alpha^{6}+150 \alpha^{4}+25 \alpha^{2}+2\right) \\
\mathrm{E}\left(X^{6}\right) & =\frac{\beta^{6}}{2}\left(10,395 \alpha^{12}+11,340 \alpha^{10}+5670 \alpha^{8}+1680 \alpha^{6}+315 \alpha^{4}+36 \alpha^{2}+2\right)
\end{aligned}
$$

\subsection{Illustrative Example II with Real-World Data}

Fleroxacin is a fluoroquinolone derivative for a broad antibacterial spectrum and powerful in vitro activity against various gram-negative and many other gram-positive varieties. This antibacterial element was studied in [32] with the aim of estimating the representative values of clearance on systemic availability $(\mathrm{D} / \mathrm{F})$ and volume of distribution on systemic availability (I/F), after administering therapeutic doses of fleroxacin. In addition, the factors that influence the disposition of fleroxacin were detected and to what degree. The individuals studied were 172 volunteers, men and women, healthy, without infections and within a wide range of ages. Among the data that were analyzed in [32] are the following measurements (in $\mathrm{ml} / \mathrm{min}$ ) of clearance on $\mathrm{D} / \mathrm{F}$ and treatinine clearance (Dcr). In this second illustration with real data, we consider a data set corresponding to the Dcr. Table 5 provides descriptive statistics of the Dcr data. Table 6 reports values of the maximum likelihood estimates, SEs, log-lik, AIC, and BIC as well as the KS $p$-values for the BBS1, BBS2, and BBS3 models. Figure 4 shows the good agreement of the BBS3 model in relation to the BBS2 and BBS2 models, such as supported by the QQ plots of Figure 5.

Table 5. Summary statistics with Dcr data.

\begin{tabular}{ccccc}
\hline $\boldsymbol{n}$ & Mean & SD & CS & CK \\
\hline 172 & 69.623 & 38.0311 & -0.2112 & 2.1637 \\
\hline
\end{tabular}

Table 6. Maximum likelihood estimates (and SEs in parenthesis) of the indicated distribution parameters with Dcr data and model selection indicators.

\begin{tabular}{crrr}
\hline Estimate/Indicator & BBS1(SE) & BBS1 (SE) & BBS3 (SE) \\
\hline$\widehat{\alpha}$ & $0.4308(0.0252)$ & $0.6976(0.0283)$ & $0.6784(0.0442)$ \\
$\widehat{\beta}$ & $27.9125(0.9800)$ & $31.7897(1.4594)$ & $15.6625(1.5963)$ \\
$\widehat{\delta}$ & $-2.5689(0.1831)$ & $-2.6272(0.3830)$ & $0.4213(0.1031)$ \\
Log-likelihood & -870.4175 & -851.217 & -838.790 \\
AIC & 1746.835 & 1708.434 & 1683.580 \\
BIC & 1756.277 & 1717.876 & 1692.602 \\
KS statistic & 0.0698 & 0.0640 & 0.0407 \\
KS $p$-value & 0.4329 & 0.4949 & 0.7521 \\
\hline
\end{tabular}




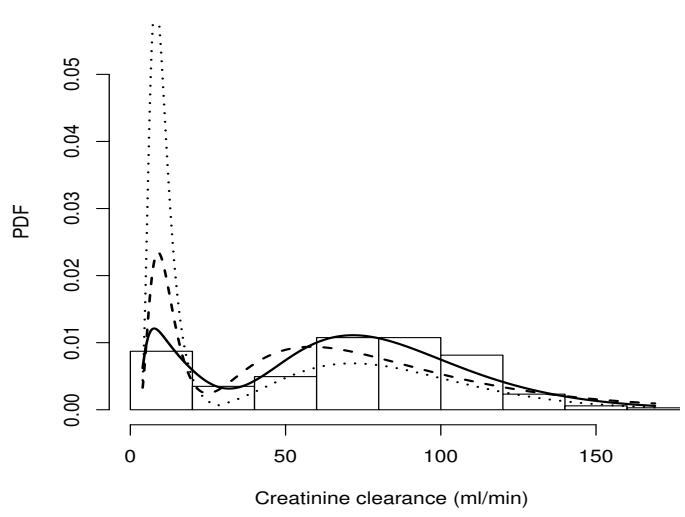

(a)

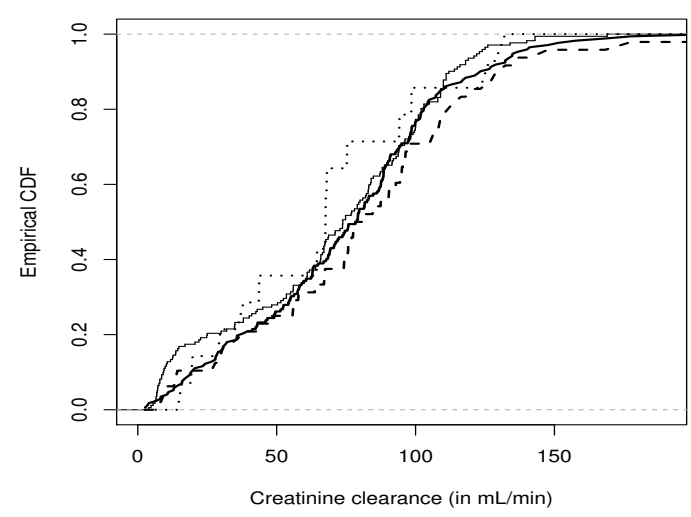

(b)

Figure 4. Histogram with estimated BBS1—dotted line, BBS2—-dashed line, and BBS3 — solid line, PDFs (a); and plots of empirical CDF versus the BBS1—dotted line, BBS2—dashed line, and BBS3 — solid line, theoretical CDFs (b) with Dcr data.

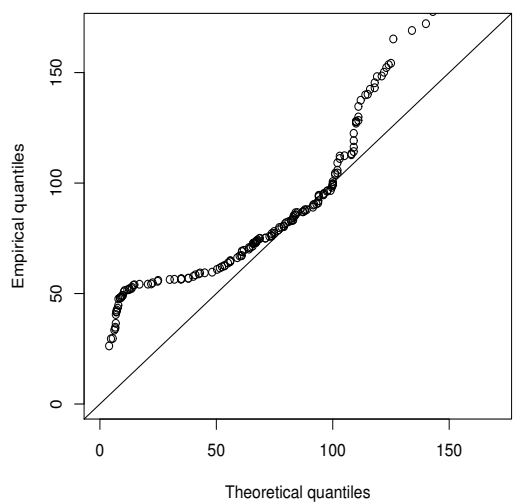

(a)

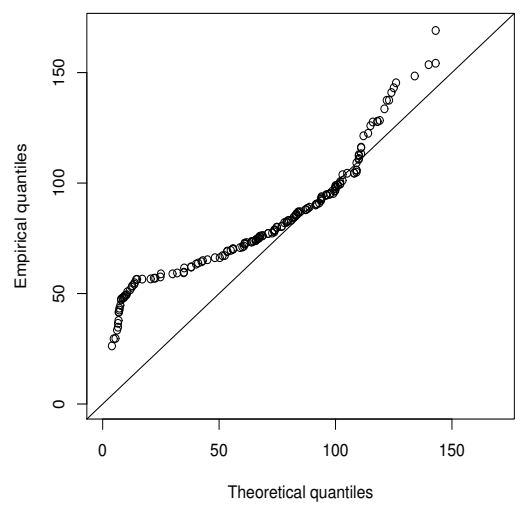

(b)

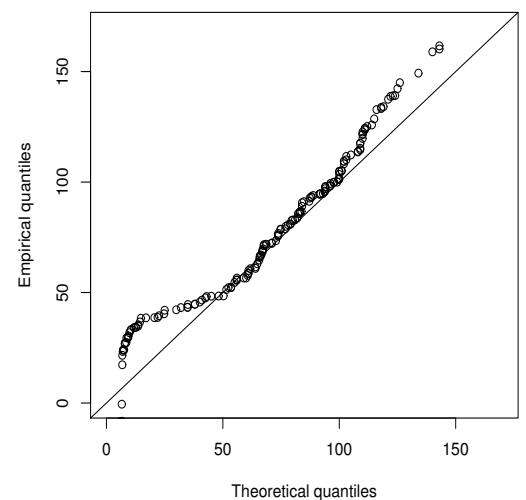

(c)

Figure 5. QQ plots of the BBS1 (a), BBS2 (b) and BBS3 (c) distributions with Dcr data.

Similarly to Example I, once the best model to describe these data has been selected, that is, the BBS3 distribution, and its parameters estimated, we obtain the mean treatinine clearance, which is $70.602 \mathrm{~mL} / \mathrm{min}$. Once again, this information is obtained from the estimates reported in Table 6 and the expressions taken from Section 3.4 specified in Example I.

\section{Conclusions, Limitations and Future Investigation}

In this paper, we have proposed a new bimodal Birnbaum-Saunders distribution, which is widely flexible. We have estimated the model parameters with the moment and maximum likelihood methods and used information criteria for model selection to assess the adequacy of the new bimodal Birnbaum-Saunders distribution in comparison with other competitor distributions.

We have conducted a Monte Carlo simulation study to empirically evaluate the statistical performance of the moment and maximum likelihood estimators of the new bimodal Birnbaum-Saunders distribution. Furthermore, we have studied coverage probabilities and the average length of confidence intervals of the corresponding parameters based on the asymptotic normality of these estimators. This simulation study reported a good statistical performance of such estimators. 
Two data analyses were conducted related to environmental and medical sciences with two competing models. Both of these analyses reported a suitable performance of the new model that was superior to all of the competing models, providing evidence that the bimodal Birnbaum-Saunders distribution is a good alternative for modeling bimodal data. Such results reported that the bimodal Birnbaum-Saunders model may be a new setting for analyzing this type of data. The new approach is an addition to the tools of applied statisticians and diverse practitioners interested in the modeling of data. From these applications, we have obtained helpful information that can be employed by practitioners and users of statistics.

Some limitations of our methodology are the instability of the maximum likelihood estimators for small samples. The bootstrap and Jackknife methods and the application of bias correction, mainly for small sample sizes, can be proposed to improve the statistical performance of these estimators. In addition, the use of explanatory variables in the modeling is of interest. Thus, their effect on the different parameters of the new bimodal Birnbaum-Saunders distribution is a topic to be studied in a future work, as detailed next. Other topics of future research based on this new distribution are related to studying how multivariate, regression, quantile, spatial, temporal, partial least squares, principal components, and sampling structures can be inserted in the modeling of bimodal data [33-44]. In addition, Tobit and Cobb-Douglas type frameworks may be analyzed in the present thematic $[45,46]$. Moreover, censored observations might be studied in the context of our investigation [7,47].

The authors are working on these and other issues associated with the present investigation, and the corresponding findings are expected to be reported in future works.

Author Contributions: Data curation, J.R., J.A.; formal analysis, J.R., J.A., V.L., C.M.-B.; investigation, J.R., J.A., V.L.; methodology, J.R., J.A., V.L., C.M.-B.; writing-original draft, J.R., J.A.; writing—review and editing, V.L., C.M.-B. All authors have read and agreed to the published version of the manuscript.

Funding: Research of J.R. and J.A. was supported by Project grant Semillero UA-2021 of the Universidad de Antofagasta, Chile. Research of V.L. was partially supported by FONDECYT, project grant number 1200525, from the National Agency for Research and Development (ANID) of the Chilean government under the Ministry of Science, Technology, Knowledge and Innovation.

Institutional Review Board Statement: Not applicable.

Informed Consent Statement: Not applicable.

Data Availability Statement: The data analyzed in this paper are available from the authors under request.

Acknowledgments: The authors would also like to thank the Editors and three Reviewers for their constructive comments which led to improving the presentation of the manuscript.

Conflicts of Interest: The authors declare no conflict of interest.

\section{References}

1. Johnson, N.L.; Kotz, S.; Balakrishnan, N. Continuous Univariate Distributions; Wiley: New York, NY, USA, 1994 ; Volume 1-2.

2. Balakrishnan, N.; Gupta, R.C.; Kundu, D.; Leiva, V.; Sanhueza, A. On some mixture models based on the Birnbaum-Saunders distribution and associated inference. J. Stat. Plan. Inference 2011, 141, 2175-2190. [CrossRef]

3. Birnbaum, Z.W.; Saunders, S.C. A new family of life distributions. J. Appl. Probab. 1969, 6, 319-327. [CrossRef]

4. Leiva, V.; Saunders, S.C. Cumulative damage models. In Wiley StatsRef: Statistics Reference Online; Wiley: Hoboken, NJ, USA, 2015; pp. 1-10.

5. Leiva, V. The Birnbaum-Saunders Distribution; Academic Press: New York, NY, USA, 2016.

6. Balakrishnan, N.; Kundu, D. Birnbaum-Saunders distribution: A review of models, analysis, and applications. Appl. Stoch. Model. Bus. Ind. 2019, 35, 4-49. [CrossRef]

7. Leao, J.; Leiva, V.; Saulo, H.; Tomazella, V. Incorporation of frailties into a cure rate regression model and its diagnostics and application to melanoma data. Stat. Med. 2018, 37, 4421-4440. [CrossRef]

8. Balakrishnan, N.; Leiva, V.; Sanhueza, A.; Vilca, F. Estimation in the Birnbaum-Saunders distribution based on scale-mixture of normals and the EM-algorithm. Stat. Oper. Res. Trans. 2009, 33, 171-192. 
9. Bhatti, C. The Birnbaum-Saunders autoregressive conditional duration model. Math. Comput. Simul. 2010, 80, $2062-2078$. [CrossRef]

10. Kotz, S.; Leiva, V.; Sanhueza, A. Two new mixture models related to the inverse Gaussian distribution. Methodol. Comput. Appl. Probab. 2010, 12, 199-212. [CrossRef]

11. Aslam, M.; Jun, C.H.; Ahmad, M. New acceptance sampling plans based on life tests for Birnbaum-Saunders distributions. J. Stat. Comput. Simul. 2011, 81, 461-470. [CrossRef]

12. Santos-Neto, M.; Cysneiros, F.J.A.; Leiva, V.; Barros, M. On a reparameterized Birnbaum-Saunders distribution and its moments, estimation and applications. REVSTAT Stat. J. 2014, 12, 247-272.

13. Marchant, C.; Leiva, V.; Cysneiros, F.J.A. A multivariate log-linear model for Birnbaum-Saunders distributions. IEEE Trans. Reliab. 2016, 65, 816-827. [CrossRef]

14. Garcia-Papani, F.; Leiva, V.; Uribe-Opazo, M.A.; Aykroyd, R.G. Birnbaum-Saunders spatial regression models: Diagnostics and application to chemical data. Chemom. Intell. Lab. Syst. 2018, 177, 114-128. [CrossRef]

15. Athayde, E.; Azevedo, A.; Barros, M.; Leiva, V. Failure rate of Birnbaum-Saunders distributions: Shape, change-point, estimation and robustness. Braz. J. Probab. Stat. 2019, 33, 301-328. [CrossRef]

16. Chaves, N.L.; Azevedo, C.L.; Vilca, F.; Nobre, J.S. A new Birnbaum-Saunders type distribution based on the skew-normal model under a centered parameterization. Chil. J. Stat. 2019, 10, 55-76.

17. Mazucheli, J.; Menezes, A.F.B.; Dey, S. The unit Birnbaum-Saunders distribution with applications. Chil. J. Stat. 2018, 9, 47-57.

18. Leiva, V.; Marchant, C.; Ruggeri, F.; Saulo, H. A criterion for environmental assessment using Birnbaum-Saunders attribute control charts. Environmetrics 2015, 26, 463-476. [CrossRef]

19. Arrué, J.; Arellano, R.; Gomez, H.W.; Leiva, V. On a new type of Birnbaum-Saunders models and its inference and application to fatigue data. J. Appl. Stat. 2020, 47, 2690-2710. [CrossRef]

20. Sanchez, L.; Leiva, V.; Galea, M.; Saulo, H. Birnbaum-Saunders quantile regression models with application to spatial data. Mathematics 2020, 8, 1000. [CrossRef]

21. Mazucheli, M.; Leiva, V.; Alves, B.; Menezes, A.F.B. A new quantile regression for modeling bounded data under a unit Birnbaum-Saunders distribution with applications in medicine and politics. Symmetry 2021, 13, 682. [CrossRef]

22. Balakrishnan, N.; Leiva, V.; López, J. Acceptance sampling plans from truncated life tests based on the generalized BirnbaumSaunders distribution. Commun. Stat. Simul. Comput. 2007, 36, 643-656. [CrossRef]

23. Marchant, C.; Leiva, V.; Cavieres, M.F.; Sanhueza, A. Air contaminant statistical distributions with application to PM10 in Santiago, Chile. Rev. Environ. Contam. Toxicol. 2013, 223, 1-31.

24. Martinez, S.; Giraldo, R.; Leiva, V. Birnbaum-Saunders functional regression models for spatial data. Stoch. Environ. Res. Risk Assess. 2019, 33, 1765-1780. [CrossRef]

25. Kannan, G.; Jeyadurga, P.; Balamurali, S. Economic design of repetitive group sampling plan based on truncated life test under Birnbaum-Saunders distribution. Commun. Stat. Simul. Comput. 2021. [CrossRef]

26. Olmos, N.M.; Martínez-Flórez, G.; Bolfarine, H. Bimodal Birnbaum-Saunders distribution with applications to non negative measurements. Commun. Stat. Theory Methods 2017, 46, 6240-6257. [CrossRef]

27. Vila, R.; Leao, J.; Saulo, H.; Nabeed, M.; Santos-Neto, M. On a bimodal Birnbaum-Saunders distribution with applications to lifetime data. Braz. J. Probab. Stat. 2020, 34, 495-518. [CrossRef]

28. Leiva, V.; Tejo, M.; Guiraud, P.; Schmachtenberg, O.; Orio, P.; Marmolejo, F. Modeling neural activity with cumulative damage distributions. Biol. Cybern. 2015, 109, 421-433. [CrossRef]

29. Elal-Olivero, D. Alpha-skew-normal distribution. Proyecciones 2010, 29, 224-240. [CrossRef]

30. Ng, H.K.T.; Kundu, D.; Balakrishnan, N. Modified moment estimation for the two-parameter Birnbaum-Saunders distribution. Comput. Stat. Data Anal. 2003, 43, 283-298. [CrossRef]

31. Azzalini, A.; Bowman, A.W. A look at some data on the old faithful geyser. J. R. Stat. Soc. C 1990, 39, 357-365. [CrossRef]

32. Reigner, B.G.; Welker, H.A. Factors influencing elimination and distribution of fleroxacin: Metaanalysis of individual data from 10 pharmacokinetic studies. Antimicrob. Agent Chemother. 1996, 40, 575-580. [CrossRef]

33. Aykroyd, R.G.; Leiva, V.; Marchant, C. Multivariate Birnbaum-Saunders distributions: Modelling and applications. Risks 2018, 6, 21. [CrossRef]

34. Puentes, R.; Marchant, C.; Leiva, V.; Figueroa-Zúñiga, J.I.; Ruggeri, F. Predicting PM2.5 and PM10 levels during critical episodes management in Santiago, Chile, with a bivariate Birnbaum-Saunders log-linear model. Mathematics 2021, 9, 645. [CrossRef]

35. Marchant, C.; Leiva, V.; Cysneiros, F.J.A.; Liu, S. Robust multivariate control charts based on Birnbaum-Saunders distributions. J. Stat. Comput. Simul. 2018, 88, 182-202. [CrossRef]

36. Saulo, H.; Leao, J.; Vila, R.; Leiva, V.; Tomazella, V. On mean-based bivariate Birnbaum-Saunders distributions: Properties, inference and application. Commun. Stat. Theory Methods 2020, 49, 6032-6056. [CrossRef]

37. Dasilva, A.; Dias, R.; Leiva, V.; Marchant, C.; Saulo, H. Birnbaum-Saunders regression models: A comparative evaluation of three approaches. J. Stat. Comput. Simul. 2020, 90, 2552-2570. [CrossRef]

38. Leiva, V.; Saulo, H.; Souza, R.; Aykroyd, R.G.; Vila, R. A new BISARMA time series model for forecasting mortality using weather and particulate matter data. J. Forecast. 2021, 40, 346-364. [CrossRef]

39. Saulo, H.; Leao, J.; Leiva, V.; Aykroyd, R.G. Birnbaum-Saunders autoregressive conditional duration models applied to highfrequency financial data. Stat. Pap. 2019, 60, 1605-1629. [CrossRef] 
40. Huerta, M.; Leiva, V.; Liu, S.; Rodriguez, M.; Villegas, D. On a partial least squares regression model for asymmetric data with a chemical application in mining. Chemom. Intell. Lab. Syst. 2019, 190, 55-68. [CrossRef]

41. Rodriguez, M.; Leiva, V.; Huerta, M.; Lillo, M.; Tapia, A.; Ruggeri, F. An asymmetric area model-based approach for small area estimation applied to survey data. REVSTAT Stat. J. 2021, 19, 399-420.

42. Costa, E.; Santos-Neto, M.; Leiva, V. Optimal sample size for the Birnbaum-Saunders distribution under decision theory with symmetric and asymmetric loss functions. Symmetry 2021, 13, 926. [CrossRef]

43. Martin-Barreiro, C.; Ramirez-Figueroa, J.A.; Nieto, A.B.; Leiva, V.; Martin-Casado, A.; Galindo-Villardón, M.P. A new algorithm for computing disjoint orthogonal components in the three-way Tucker model. Mathematics 2021, 9, 203. [CrossRef]

44. Martin-Barreiro, C.; Ramirez-Figueroa, J.A.; Cabezas, X.; Leiva, V.; Galindo-Villardón, M.P. Disjoint and functional principal component analysis for infected cases and deaths due to COVID-19 in South American countries with sensor-related data. Sensors 2021, 21, 4094. [CrossRef] [PubMed]

45. Desousa, M.; Saulo, H.; Leiva, V.; Scalco, P. On a tobit-Birnbaum-Saunders model with an application to medical data. J. Appl. Stat. 2018, 45, 932-955. [CrossRef]

46. de La Fuente-Mella, H.; Rubilar, R.; Chahuan-Jimenez, K.; Leiva, V. Modeling COVID-19 cases statistically and evaluating their effect on the economy of countries. Mathematics 2021, 9, 1558. [CrossRef]

47. Azevedo, C.; Leiva, V.; Athayde, E.; Balakrishnan, N. Shape and change point analyses of the Birnbaum-Saunders-t hazard rate and associated estimation. Comput. Stat. Data Anal. 2012, 56, 3887-3897. [CrossRef] 